\title{
Energy Management and Drivability Control Problems for Hybrid Electric Vehicles
}

\author{
Pierluigi Pisu, Kerem Koprubasi and Giorgio Rizzoni, Fellow, IEEE
}

\begin{abstract}
Control strategies for hybrid-electric vehicles generally target several simultaneous objectives. The primary one is the minimization of the vehicle fuel consumption, while also attempting to minimize emissions and to maintain or enhance drivability. Hybrid electric vehicle improvements in fuel economy and emissions strongly depend on the energy management strategy and vehicle powertrain configuration. This paper presents an overview of state-of-the-art energy management approaches for the control of different hybrid electric powertrain configurations.
\end{abstract}

\section{INTRODUCTION}

The gas price increase in the last few years and the pressure to become less dependable from foreign oil is driving the market of passenger vehicle and heavy duty trucks towards hybrid electric solutions that allow high fuel economy, low emissions and enhanced drivability. As a result, there is an increasing research interest in efficient hybrid electric powertrain configurations and in the design of corresponding control strategies. Depending on the particular application, some powertrain topologies may be more suitable and appropriate than others by providing better fuel economy and drivability. Choice of the appropriate topology requires preliminary study of driving cycles, vehicle size and weight, desired performance, and on-road/off-road application. Once the topology has been selected, the design of an effective energy management control strategy is an essential key for an efficient hybrid electric vehicle (HEV).

At any time and for any vehicle speed, the control strategy has to determine the power distribution between primary energy converter (FC) and renewable electrical storage system (RESS), as well as the optimal gear ratio of the transmission, if any. Regardless of the vehicle topology, the primary objective of any control strategy is to satisfy the driver's power demand by managing the power flows from the various energy storage devices to minimize fuel consumption and simultaneously satisfying other constraints such as regulation of the RESS state of charge (SOC) or state of energy (SOE), emissions and drivability.

In order to meet these requirements, many optimal control strategies for HEVs have been proposed in the past. In particular, they can be classified in three groups: dynamic

P. Pisu is with the Center for Automotive Research, The Ohio State University, 930 Kinnear Rd.,Columbus, OH 43212. USA.

K. Koprubasi and G. Rizzoni are with the Department of Mechanical Engineering and Center for Automotive Research, The Ohio State University, 930 Kinnear Rd.,Columbus, OH 43212. USA (e-mail: rizzoni.1@osu.edu, koprubasi.1@osu.edu ). (Corresponding author phone: 614-688-3856; fax: 614-688-4111; e-mail:rizzoni.1@osu.edu ). programming approaches as in [1], intelligent control techniques such as rule based, fuzzy logic [2] and neural networks [3], and methods based on the conversion of the electric power into equivalent fuel consumption [4],[5]. Unfortunately, these approaches have some major drawbacks like the necessity to know the driving cycle a priori and the difficulty in finding an analytical expression for the controller [1]-[3]. It follows that, in practical applications, the real behavior of the system will differ even significantly from the optimal solution.

In this paper, an overview of control problems for different vehicle topologies with two or more DOF is presented. The control problems are cast in a general framework independent on the topology. A revised and extended version of the equivalent control minimization strategy (ECMS) proposed by Paganelli [4],[5] and by Pisu $[6],[7]$ is then used to solve the energy consumption minimization problem. The paper also describes the problem of control for drivability in power split systems [8],[9] illustrating a solution based on the combination of the ECMS strategy with traditional linear/non-linear controls. Details of this solution are reported in [9].

The paper is organized as follows. In Section II different hybrid electric vehicle topologies are illustrated, whereas in Section III the energy management problem is explained. Section IV deals with the explanation of the proposed equivalent control minimization strategy for 2-DOF and 3DOF systems. In Section V, the control policy is extended to consider the drivability aspect.

\section{HYBRID ELECTRIC VEHICLE TOPOLOGIES}

In Fig. 1, a parallel hybrid powertrain configuration for a passenger car is depicted. An internal combustion engine (ICE) is coupled to an electric motor (EM) in a pretransmission configuration. A renewable energy storage system (RESS) constituted in general by batteries or supercapacitors provide the necessary energy storage for the electric motor to function either as motor or generator.

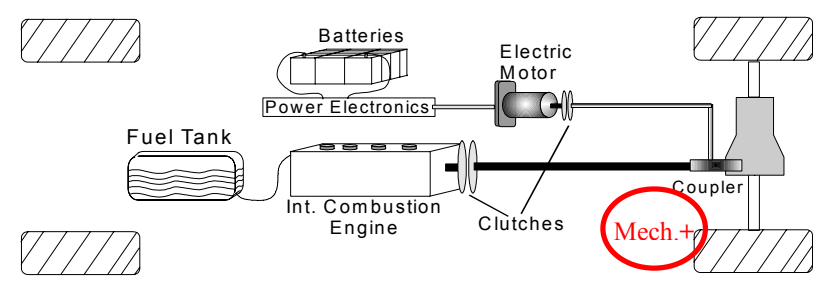

Fig. 1. Parallel hybrid configuration. 


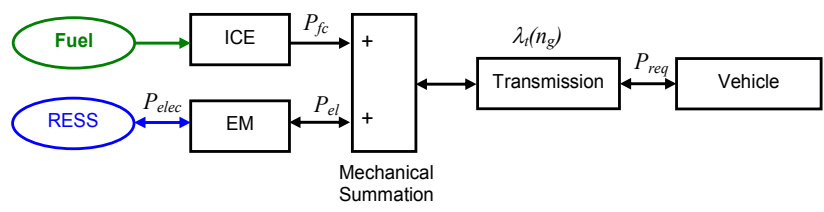

Fig. 2. Schematic representation of a parallel hybrid with pre-transmission mechanical coupling.

Figures 2 and 3 represent two possible schematic representations of parallel hybrid configurations: the first with pre-transmission mechanical coupling, and the second with post-transmission mechanical coupling. In this case, the power summation node is a mechanical summation node, i.e.

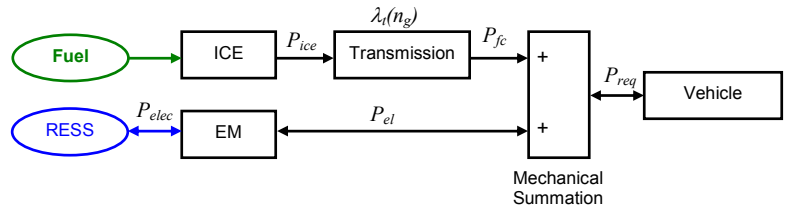

Fig. 3. Schematic representation of a parallel hybrid with post-transmission mechanical coupling.

power summation is obtained by addition of the mechanical torque from ICE engine $P_{f c}$ and electric machine $P_{e l}$. So, instead of reasoning in terms of power, it is possible, without loss of generality, to reason in terms of torque, and rewrite the power constraint $P_{r e q}=P_{f c}+P_{e l}$ as

$$
\begin{aligned}
& T_{\text {req }}=\lambda_{t}\left(n_{g}\right) \eta_{t}\left(T_{\text {ice }}+\lambda_{b} \bar{\eta}_{b} \cdot T_{e m}\right) \text { for pre-transmission } \\
& T_{\text {req }}=\lambda_{t}\left(n_{g}\right) \eta_{t} T_{\text {ice }}+\lambda_{b} \bar{\eta}_{b} \cdot T_{\text {em }} \text { for post-transmission }
\end{aligned}
$$

where $\lambda_{t}\left(n_{g}\right)$ is the gear ratio function of the gear number, $\lambda_{b}$ is the gear ratio of the mechanical coupling, $\eta_{t}$ is the transmision efficiency, $T_{i c e}$ is the torque produced by the engine, $T_{e m}$ is the torque produced by the electric motor, $T_{r e q}$ is the requested torque from the driver, and

$$
\bar{\eta}_{b}= \begin{cases}\eta_{b} & \text { if } T_{e m} \geq 0 \\ \frac{1}{\eta_{b}} & \text { if } T_{e m}<0\end{cases}
$$

with $\eta_{b}$ efficiency of the mechanical coupling. In most recent developments, it is possible to have a RESS connected to more than one electric motor (EM).

For the case of series hybrid, a schematic picture is shown in Fig. 4 and 5. In this case, the power summation node is an electrical summation node, i.e. power summation is obtained by addition of the electric power from generator and the electric storage.

In addition to the topologies illustrated, we have at least two different RESS architectures. The first architecture is depicted in Fig. 6 where there is a dedicated DC/DC converter for each power source. The second one instead has only one $\mathrm{DC} / \mathrm{DC}$ converter for one of the two sources (Supercaps) while the other one is directly connected to the electric bus.

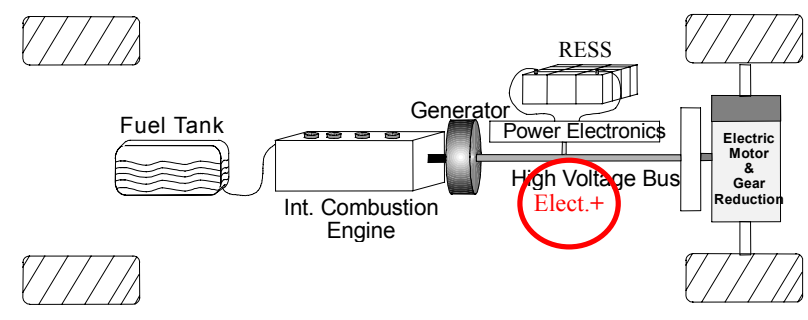

Fig. 4. Series hybrid configuration.

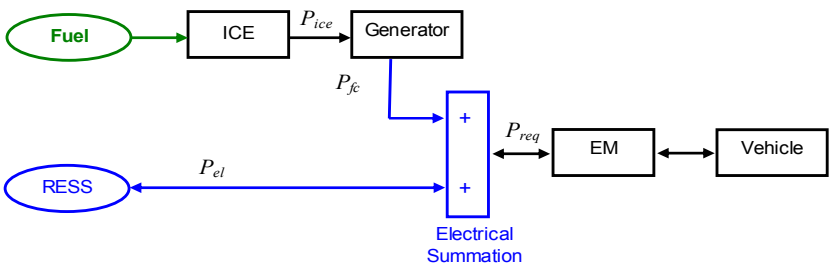

Fig. 5. Schematic representation of a series hybrid topology.

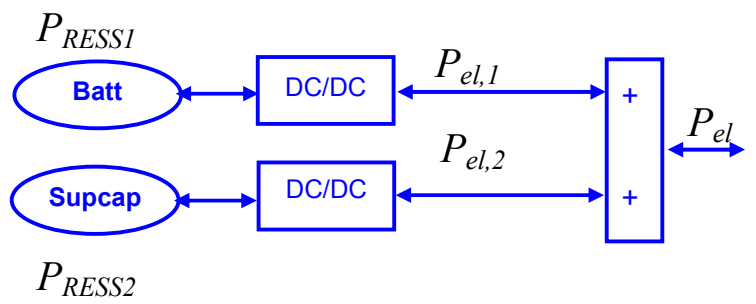

Fig. 6. Schematic representation of the connection of two electrical power sources: configuration 1.

In series hybrid powertrain, additional fuel converter configurations are also available. In particular, we consider two additional architectures. The first is constituted by two engines and one generator as illustrated in Fig. 7. The two engines are coupled in such a way that they are counter rotating. One engine is connected to the rotor of the generator while the other one is connected to the stator. In this condition, the torque is the same while the speed is added. From a control point of view, one engine will be always running while the second one will be used when additional power is need. Sizing of the engines is very important, as well as appropriate control to guarantee maximum efficiency engine operation.

The second architecture instead is constituted by two gensets where the generator can be both synchronous or one of them can be asynchronous (Fig. 8).

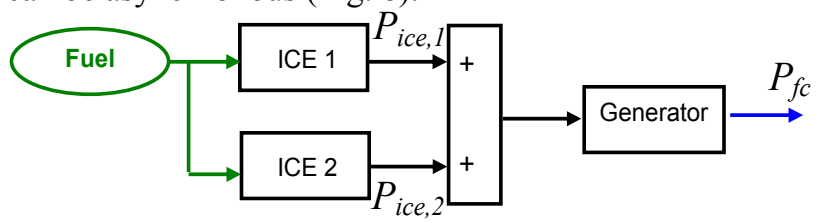

Fig. 7. Schematic representation of the connection of two engines and one generator.

In the case of synchronous generators, the two gensets must have same speed and phase as to be synchronized. If instead one of the two is asynchronous, then the corresponding 
genset must run at a slightly higher speed than the other one depending on the slip conditions.

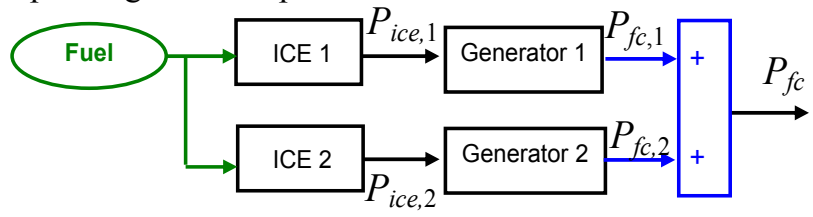

Fig. 8. Schematic representation of the connection of two gensets.

Finally, there exists another topology called "power split", which is very common in commercial passenger cars (e.g. Ford Escape HEV) and constitutes a combination of parallel and series topologies (Fig. 9).

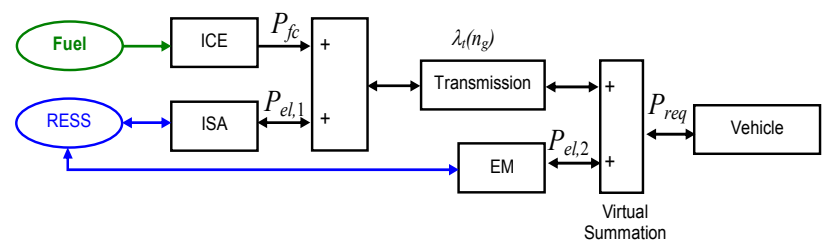

Fig. 9. Schematic representation of power split topology.

This topology is constituted by an engine coupled to an integrated starter-alternator (ISA) and a second electric motor (EM) which maybe connected to the rear axle of the vehicle, and then mechanically decoupled from the rest of the powertrain. Both ISA and EM are connected to the same RESS. With this configuration, the powertrain can function in parallel pre-transmission mode (ICE+ISA), posttransmission mode (ICE+EM), series mode (ICE+ISA in regeneration mode and EM in motoring mode), and anything in between.

\section{ENERGY MANAGEMENT PROBLEM}

The basic challenge of energy management in a hybrid electric vehicle is to assure optimal use and regeneration of the total energy in the vehicle. Regardless of the powertrain configuration, at any time and for any vehicle speed, the control strategy has to determine the power distribution between primary energy converter (FC) and renewable electrical storage system (RESS) [4]-[7]. When two storage systems or two fuel converters are available additional power distribution between the RESSs and between the fuel converters has to be determined. These decisions are constrained by two factors. First of all, the motive power requested by the driver must always be satisfied up to a known limit (maximum power demand). Secondly, the state of charge of the RESS must be maintained within preferred limits, allowing the vehicle to be charge sustaining. Within these constraints, the first objective is to operate the powertrain in order to achieve the maximum fuel economy. Ideally the motive power must be split at each time to minimize the overall fuel consumption over a given trip, such as

$$
\min _{\left\{P_{f c, i}(t), P_{e l, i}(t): i=1,2\right\}} \int_{0}^{T} \dot{m}_{f}(\tau) d \tau
$$

with the constraints

$$
\begin{aligned}
& P_{r e q}(t)=P_{f c}(t)+P_{e l}(t) \\
& \quad=P_{f c, 1}(t)+P_{f c, 2}(t)+P_{e l, 1}(t)+P_{e l, 2}(t) \quad \forall t \\
& 0<S O E_{i, \min } \leq S O E_{i} \leq S O E_{i, \max } \leq 1 \\
& 0 \leq P_{f c, i}(t) \leq P_{f c, i_{\max }} \\
& P_{e l, i_{\min }} \leq P_{e l, i}(t) \leq P_{e l, i_{\max }} \quad i=1,2
\end{aligned}
$$

where $T$ is the duration of the trip, $\dot{m}_{f}(t)$ is the fuel flow rate at time $t, P_{e l, i}(t)$ is the power provided by the $i^{\text {th }}$ electrical accumulator at time $t, P_{f c, i}(t)$ is the power provided by the $i^{\text {th }}$ fuel converter (engine only or engine plus generator depending on the configuration) at time $t, P_{\text {req }}$ is the power requested by the driver and $S O E_{i}$ is the state of energy of the RESS ${ }_{\mathrm{i}}$ and it is related to the energy or charge accumulated in the RESS (i.e. $S O E_{i}$ is an indicator of how much energy can be stored or withdrawn from the RESS $\mathrm{i}_{\mathrm{i}}$ ).

The main problem with this approach is that in order to solve such an optimization problem the whole driving schedule has to be known a priori, thus real-time control cannot be readily implemented. To avoid this drawback, one can replace the global criterion by a local one, reducing the problem to a minimization of the equivalent fuel consumption at each time [4], [5]. The local criteria becomes at all times

$$
\min _{\left\{P_{f c, i}(t), P_{e l, i}(t): i=1,2\right\}} \dot{m}_{f, e q}(t) \quad \forall t
$$

where $\dot{m}_{f, e q}(t)$ is the equivalent fuel flow rate at time $t$ and with the same constraints as before. The equivalent fuel cost $\dot{m}_{f, e q}(t)$ is the sum of the actual fuel consumption rate of the fuel converter, $\dot{m}_{f c}(t)$, and the equivalent fuel use of the RESS, $\dot{m}_{f, R E S S, e q}(t)$. The global minimization problem represented in (1) and the local minimization shown in (2) are not strictly equivalent. However, local minimization results in a formulation amenable to real-time control, while the use of the equivalent fuel flow rate indirectly accounts for the non-local nature of the problem.

\section{EQUIVALENT CONTROL MINIMIZATION STRATEGY}

The equivalent fuel consumption minimization strategy is based on the assumption of quasi-static behavior of the system. In general, for a normal vehicle, this behavior is 
characterized by capturing phenomena that are in the order of 0.5-1 sec, while faster dynamics are neglected. The main idea consists in assigning future fuel savings and costs to the actual use of electric energy, and in particular:

- a present discharge of the RESS corresponds to a future fuel consumption that will be necessary to recharge the RESS;

- a present battery charge corresponds to a future fuel savings because this energy will be available in the future to be used at a lower cost.

The strategy is charge sustaining because balances the costs in the future with the savings in the future. The equivalent fuel consumption is generally defined as

$$
\dot{m}_{f, e q}=\dot{m}_{f, I C E}\left(\omega_{i c e}, P_{i c e}\right)+\dot{m}_{f, R E S S, e q}\left(P_{e l}\right)
$$

where $\omega_{i c e}$, is the engine speed, and $P_{i c e}$, is the power provided by the engine.

In the following sections we distinguish between a 2-DOF system where there is a single power split (single fuel converter and single RESS), and systems with higher DOF where splits among RESSs and fuel converters are considered.

\section{A. Equivalent fuel consumption for 2-DOF systems}

In the following, we will consider discharging and charging condition separately. With the term "recharging conditions" we intend the time intervals when there is an energy flow from the fuel converter to the RESS, while with the term "recovering conditions" we intend the time intervals when there is an energy flow from the wheels to the RESS.

\section{1) Discharging mode}

The amount of energy $\Delta E_{\text {RESS,dis }}$ removed from the RESS at a given power $P_{e l}$ during an interval $\Delta t$ is

$$
\Delta E_{R E S S, d i s}=\Delta t P_{R E S S, d i s}=\frac{\Delta t P_{e l}}{\eta_{e l, d i s}\left(P_{e l}\right)}
$$

where for the parallel hybrid

$$
\eta_{e l, d i s}\left(P_{e l}\right)=\eta_{b} \eta_{e m, d i s}\left(P_{e l}\right) \eta_{R E S S, d i s}\left(P_{e l}\right)
$$

with $\eta_{b}$ efficiency of the mechanical coupling, $\eta_{e m, d i s}$ efficiency of the electric motor at discharging, and $\eta_{\text {,RESS, dis }}$ efficiency of the electric storage at discharging, whereas for the series hybrid

$$
\eta_{e l, d i s}\left(P_{e l}\right)=\eta_{p e, d i s}\left(P_{e l}\right) \eta_{R E S S, d i s}\left(P_{e l}\right)
$$

with $\eta_{p e, d i s}$ efficiency of the power electronics that connects the electric storage to the electric bus during discharging. The future cost of $\Delta E_{R E S S, d i s}$ is

$$
c_{\Delta E, \text { dis }}=C_{\text {tot,chg }} \frac{\Delta E_{R E S S, d i s}}{E_{\text {tot }, \text { chg }}} \quad[g]
$$

where $E_{\text {tot,chg }}$ is the total energy recharged in the future, $C_{\text {tot,chg }}$ is the cost of $E_{\text {tot,chg }}$. This cost is a fraction of the cost of the total energy recharged into the RESS.
The cost of the total energy recharged in the future is

$$
C_{\text {tot, chg }}=\frac{E_{t o t, T A N K-R E S S}}{Q_{L H V}}=\frac{1}{Q_{L H V}} \int_{\begin{array}{c}
\text { all fiture } \\
\text { rechy.cond. }
\end{array}} \frac{\left|P_{R E S S, \text { chg }}(t)\right|}{\eta_{f c}(t) \eta_{e l}(t)} d t \quad[g]
$$

$\eta_{f c}(t)= \begin{cases}\eta_{i c e}(t) & \text { for pre - transmission parallel hybrid } \\ \eta_{t} \eta_{i c e}(t) & \text { for post - transmission parallel hybrid } \\ \eta_{\text {genset }}(t) & \text { for series hybrid }\end{cases}$

$\eta_{e l}(t)=\left\{\begin{array}{c}\eta_{b} \eta_{e m}(t) \eta_{R E S S}(t) \text { for parallel hybrid } \\ \eta_{p e}(t) \eta_{\text {RESS }}(t) \text { for series hybrid }\end{array}\right.$

where $E_{t o t, T A N K-R E S S}$ is the total energy flowing from the tank to the RESS, $Q_{L H V}$ is the low heating value of the fuel, $P_{f c, R E S S}$ is the power from the fuel converter to the RESS, and $\eta_{\text {genset }}$ is the combined efficiency of ICE and generator.

By substituting the above expressions into (5) we have

$$
c_{\Delta E, \text { dis }}=\frac{\int_{\begin{array}{c}
\text { all future } \\
\text { rechy.cond. }
\end{array}} \frac{\left|P_{R E S S, \text { chg }}(t)\right|}{\eta_{f c}(t) \eta_{e l}(t)} d t}{\int_{\substack{\text { all future } \\
\text { rechy.+recov. cond. }}}\left|P_{R E S S, \text { chg }}(t)\right| d t} \cdot \frac{1}{\eta_{e l, d i s}\left(P_{e l}\right)} \cdot \frac{P_{e l}}{Q_{L H V}} \Delta t
$$

Approximating the efficiencies by their average values, we can write

$$
c_{\Delta E, \text { dis }} \approx \frac{1}{\bar{\eta}_{f c, \text { rechg }} \bar{\eta}_{\text {el, rechg }}} \cdot \frac{1}{1+\bar{R}_{\text {recov } / \text { rech }}} \cdot \frac{1}{\eta_{e l, \text { dis }}\left(P_{e l}\right)} \cdot \frac{P_{e l}}{Q_{L H V}} \Delta t
$$

where $\bar{\eta}_{f c \text {,rechg }}$ is the average efficiency of the fuel converter during recharge, $\bar{\eta}_{e l, \text { rechg }}$ is the average efficiency of the electric path during recharge, and $\bar{R}_{\text {recov/rech }}$ is the average value of

$$
R_{\text {recov/rech }}=\frac{\int_{\begin{array}{c}
\text { all future } \\
\text { recov.cond. }
\end{array}}\left|P_{R E S S, \text { chg }}(t)\right| d t}{\int\left|P_{R E S S, \text { chg }}(t)\right| d t}
$$

The cost in terms of fuel of discharging the RESS, i.e. the equivalent fuel consumption of the RESS in discharging mode, is

$$
\dot{m}_{f, \text { RESS }, e q}=\frac{c_{\Delta E, d i s}}{\Delta t} \approx \frac{1}{\bar{\eta}_{\text {rechg }}} \cdot \frac{1}{\eta_{e l, d i s}\left(P_{e l}\right)} \cdot \frac{P_{e l}}{Q_{L H V}}
$$

with 


$$
\begin{aligned}
& \bar{\eta}_{\text {rechg }}=\bar{\eta}_{f c, \text { rechg }} \bar{\eta}_{\text {el }, \text { rechg }} \cdot\left(1+\bar{R}_{\text {recov / rech }}\right) \\
& \bar{\eta}_{x, \text { rechg }}=\frac{1}{T_{\text {rechg }}} \int_{\begin{array}{l}
\text { all future } \\
\text { rechy . cond }
\end{array}} \eta_{x}(t) d t
\end{aligned}
$$

where $T_{\text {rechg }}$ is the future time spent in recharge mode.

\section{2) Charging mode}

The amount of energy added to the RESS at a given power $P_{e l}$ during an interval $\Delta t$ is

$$
\Delta E_{R E S S, \text { chg }}=\Delta t P_{R E S S, \text { chg }}=\eta_{e l, c h g}\left(P_{e l}\right) P_{e l} \Delta t
$$

where for the parallel hybrid

$$
\eta_{e l, c h g}\left(P_{e l}\right)=\eta_{b} \eta_{e m, c h g}\left(P_{e l}\right) \eta_{R E S S, c h g}\left(P_{e l}\right)
$$

with $\eta_{b}$ efficiency of the mechanical coupling, $\eta_{e m, c h g}$ efficiency of the electric motor during charging, and $\eta_{\text {,RESS, chg }}$ efficiency of the electric storage during charging, while for the series hybrid

$$
\eta_{e l, c h g}\left(P_{e l}\right)=\eta_{p e, c h g}\left(P_{e l}\right) \eta_{R E S S, c h g}\left(P_{e l}\right)
$$

with $\eta_{p e, c h g}$ efficiency of the power electronics that connects the electric storage to the electric bus during charging.

The future saving (negative cost) associated with $\Delta E_{R E S S, \text { chg }}$ is

$$
s_{\Delta E, c h g}=S_{t o t, d i s} \frac{\Delta E_{R E S S, c h g}}{E_{t o t, d i s}} \quad[g]
$$

where $E_{\text {tot,dis }}$ is the total energy discharged in the future, $S_{t o t, d i s}$ is the saving due $E_{t o t, d i s}$. This saving is a fraction of the savings due to the total energy discharged from the RESS.

The savings due to the total energy discharged in the future is

$$
S_{\text {tot,dis }}=\frac{E_{t o t, R E S S-T A N K}}{Q_{L H V}}=\frac{1}{Q_{L H V}} \int_{\begin{array}{c}
\text { all future } \\
\text { dis. cond. }
\end{array}} \frac{\eta_{e l}(t) P_{R E S S, d i s}(t)}{\eta_{f c}(t)} d t
$$

where $E_{t o t, R E S S-T A N K}$ is the total energy flowing from the RESS to the tank (virtual).

Approximating the efficiencies by their average values, we can write

$$
s_{\Delta E, d i s} \approx \frac{\bar{\eta}_{e l, d i s}}{\bar{\eta}_{f c, d i s}} \cdot \eta_{e l, c h g}\left(P_{e l}\right) \cdot \frac{P_{e l}}{Q_{L H V}} \Delta t
$$

where $\bar{\eta}_{f c \text {,rechg }}$ is the average efficiency of the fuel converter during recharge, and $\bar{\eta}_{e l, \text { rechg }}$ is the average efficiency of the electric path during recharge.

The cost in terms of fuel of charging the RESS, i.e. the equivalent fuel consumption of the RESS in charging mode, is

$$
\dot{m}_{f, R E S S, e q}=\frac{s_{\Delta E, d i s}}{\Delta t} \approx \frac{1}{\bar{\eta}_{d i s}} \cdot \eta_{e l, c h g}\left(P_{e l}\right) \cdot \frac{P_{e l}}{Q_{L H V}}
$$

with

$$
\bar{\eta}_{\text {dis }}=\frac{\bar{\eta}_{f c, d i s}}{\bar{\eta}_{e l, d i s}}, \quad \bar{\eta}_{x, \text { dis }}=\frac{1}{T_{\text {dis }}} \int_{\begin{array}{c}
\text { all future } \\
\text { dis. cond. }
\end{array}} \eta_{x}(t) d t
$$

where $T_{\text {dis }}$ is the future time spent in discharge mode.

Summarizing, we can write the expression for the equivalent fuel consumption of the RESS as

$$
\dot{m}_{f, R E S S, e q}=\left(\gamma \frac{1}{\bar{\eta}_{\text {rechg }}} \cdot \frac{1}{\eta_{e l, d i s}\left(P_{e l}\right)}+(1-\gamma) \frac{1}{\bar{\eta}_{\text {dis }}} \cdot \eta_{e l, c h g}\left(P_{e l}\right)\right) \cdot \frac{P_{e l}}{Q_{L H V}}
$$

where $\gamma=1$ for $P_{e l} \geq 0$ and $\gamma=0$ otherwise.

In practical situations, the efficiencies $\bar{\eta}_{d i s}$ and $\bar{\eta}_{\text {rechg }}$ are considered as unknown parameters to be tuned or estimated.

\section{B. Equivalent fuel consumption for 3-DOF systems or more}

For a vehicle with two engines (Fig. 7) or two gensets (Fig. 8 ), the equivalent minimization function given by (3) becomes

$$
\dot{m}_{f, e q}=\left\{\begin{array}{l}
\dot{m}_{f, I C E 1}\left(\omega_{i c e, 1}, P_{i c e, 1}\right)+ \\
\dot{m}_{f, R E S S_{e q}}\left(P_{e l}\right) \quad \text { if } P_{r e q} \leq \bar{P}_{i c e, 1} \\
\dot{m}_{f, I C E 1}\left(\omega_{i c e 1}, P_{i c e, 1}\right)+\dot{m}_{f, I C E 2}\left(\omega_{i c e, 2}, P_{i c e, 2}\right) \\
+\dot{m}_{f, R E S S_{e q}}\left(P_{e l}\right) \quad \text { if } P_{r e q}>\bar{P}_{i c e, 1}
\end{array}\right.
$$

where $\bar{P}_{i c e, 1}$ is some power level for ICE1 that depends on the design parameters.

In order to illustrate how the previous equations for the 2DOF system can be extended, we will consider the case of a series hybrid with two RESSs. Results for the parallel hybrid cases can be obtained in a similar way. In the case of two RESSs (Fig. 6), the equivalent fuel consumption given by (3) becomes

$$
\begin{aligned}
\dot{m}_{f, e q} & =\dot{m}_{f, I C E}\left(\omega_{i c e}, P_{i c e}\right) \\
& +\dot{m}_{f, \text { RESS } 1_{e q}}\left(P_{e l, 1}\right)+\dot{m}_{f, \text { RESS } 2_{e q}}\left(P_{e l, 2}\right)
\end{aligned}
$$

As in (5) and (8), it is possible to associate to each RESS a cost of discharging and a saving of charging of the form

$$
\begin{aligned}
& c_{\Delta E, i, d i s}=C_{i, t o t, c h g} \frac{\Delta E_{R E S S, i, d i s}}{E_{i, t o t, c h g}} \quad[g] \\
& s_{\Delta E, i, c h g}=S_{i, t o t, d i s} \frac{\Delta E_{R E S S, i, c h g}}{E_{i, t o t, d i s}} \quad[g]
\end{aligned}
$$

where $\Delta E_{R E S S, i, d i s}$ is the amount of energy removed from $\operatorname{RESS}_{i}(i=1,2), \Delta E_{R E S S, i, c h g}$ is the amount of energy added to $\operatorname{RESS}_{i}, E_{i, t o t, c h g}$ is the total energy recharged in the future 
to $\mathrm{RESS}_{i}, C_{i, t o t, c h g}$ is the cost of $E_{i, \text { tot, chg }}, E_{i, t o t, d i s}$ is the total energy discharged in the future by $\operatorname{RESS}_{i}, S_{i, t o t, d i s}$ is the saving due $E_{i, t o t, d i s}$. After some manipulations and approximating the efficiencies by their average values, it can be shown that during discharging

$$
\dot{m}_{f, R E S S, i_{e q}}=\frac{c_{\Delta E, i, d i s}}{\Delta t} \approx \frac{1}{\bar{\eta}_{\text {rechg }, i}} \cdot \frac{1}{\eta_{e l, i, d i s}\left(P_{e l, i}\right)} \cdot \frac{P_{e l, i}}{Q_{L H V}}
$$

where $\bar{\eta}_{\text {rechg }, i}$ is some average recharging efficiency, and $\eta_{e l, i, d i s}\left(P_{e l, i}\right)=\eta_{p e, i, d i s}\left(P_{e l, i}\right) \eta_{R E S S, i, d i s}\left(P_{e l, i}\right)$

with $\eta_{p e, i, d i s}$ efficiency of the power electronics that connects the electric storage to the electric bus during discharging, and $\eta_{\text {,RESS,i,dis }}$ efficiency of the electric storage $\mathrm{RESS}_{\mathrm{i}}$ at discharging. During charging conditions

$$
\dot{m}_{f, R E S S, i_{e q}}=\frac{s_{\Delta E, i, d i s}}{\Delta t} \approx \frac{1}{\bar{\eta}_{d i s, i}} \cdot \eta_{e l, i, c h g}\left(P_{e l, i}\right) \cdot \frac{P_{e l, i}}{Q_{L H V}}
$$

where $\bar{\eta}_{d i s, i}$ is some average discharging efficiency, and

$$
\eta_{e l, i, c h g}\left(P_{e l, i}\right)=\eta_{p e, i, c h g}\left(P_{e l, i}\right) \eta_{R E S S, i, c h g}\left(P_{e l, i}\right)
$$

with $\eta_{p e, i, c h g}$ efficiency of the power electronics that connects the electric storage $\mathrm{RESS}_{\mathrm{i}}$ to the electric bus during charging and $\eta_{\text {,RESS,i,chg }}$ efficiency of the electric storage $\mathrm{RESS}_{\mathrm{i}}$ during charging.

\section{CONTROL FOR DRIVABILITY}

\section{A. Drivability Problem}

One of the primary objectives of the control strategy is to assure acceptable drivability of the vehicle. Several metrics have been proposed to quantify the drivability of a hybrid vehicle [8], [9]. The vast majority of these drivability metrics focus on achieving smooth gear shifting and minimizing excessive driveline vibrations.

\section{B. Dynamic Driveline Model}

The drivability control model that is explained here is an extension of the earlier work presented in [9]. The dynamics model is formulated for a specific power-split hybrid driveline configuration. The driveline incorporates a traction electric motor which drives the electric rear axle, and an ICE and an integrated started-alternator (ISA) mechanically coupled on the front axle. The representative architecture was previously shown in Fig. 9.

The details of the driveline model can be seen in Fig. 10. A nonlinear lumped parameter dynamic model is obtained using the method of Lagrange:

$$
\begin{gathered}
\left(J_{I C E}+J_{I S A}\right) \dot{\omega}_{I C E}=-b_{I C E} \omega_{I C E}+T_{I C E}+T_{I S A} \\
-T_{T C_{-} P}\left(\omega_{I C E}, \omega_{T R}\right) \\
J_{T R} \dot{\omega}_{T R}=T_{T C_{-} T}\left(\omega_{I C E}, \omega_{T R}\right) / \tau_{T R}(g)-T_{F}
\end{gathered}
$$

$$
\begin{aligned}
& J_{E M} \dot{\omega}_{E M}=T_{E M}-b_{E M} \omega_{E M}-T_{R} \tau_{G B} \\
& m_{V E H} \dot{v}_{V E H}=\frac{T_{F}}{r_{F}}+\frac{T_{R}}{r_{R}}-T_{B_{-} F}-T_{B_{-} R}-F_{R D}
\end{aligned}
$$

where $J_{*}$ represent various inertias, $T_{*}$ are torques at different locations of the driveline, $\omega_{*}$ are speeds, $\tau_{T R}(g)$ and $\tau_{G B}$ are the gear ratios for the transmission and the electric machine gearbox $\left(\tau_{T R}(g)\right.$ is a function of $\mathrm{g}=\{0,1 . .5\}$, gear index $), b_{*}$ are viscous friction coefficients, $r_{*}$ are wheel radii, $T_{B} *$ are brake torques (subscripts ${ }_{\mathrm{F}}$ and $\mathrm{R}_{\mathrm{R}}$ denote front and rear wheels), $m_{V E H}$ is the vehicle mass, $v_{V E H}$ is the vehicle speed and $F_{R D}$ is the air drag. The engine torque is transmitted to the front axle via a torque converter (TC). Expressions for the loads at the front, $T_{F}$ and the rear, $T_{R}$ wheels are given as

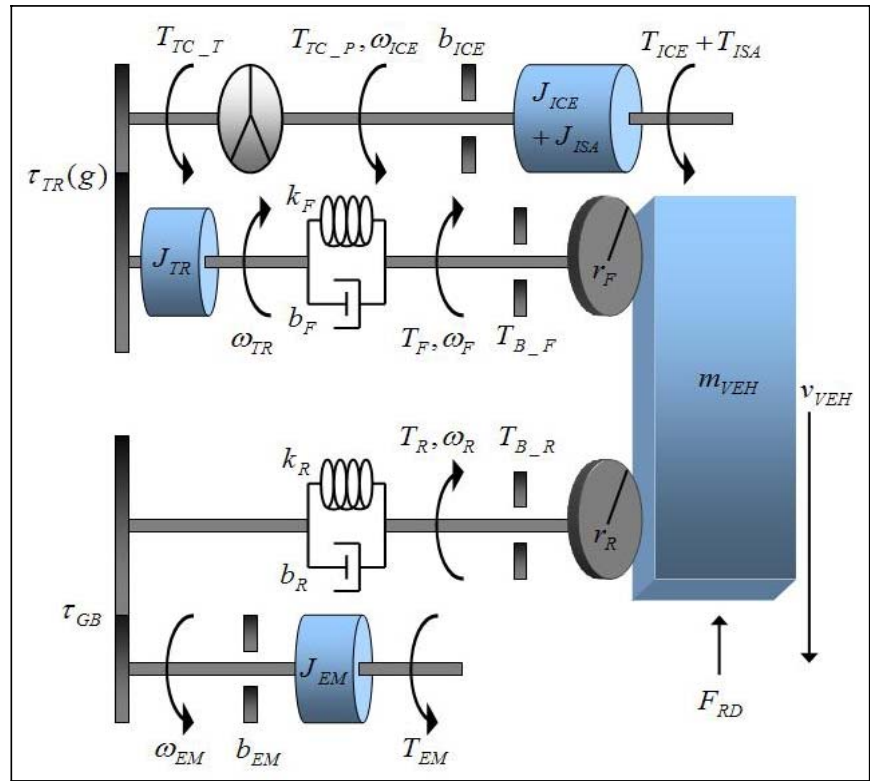

Fig. 10 Representative Driveline Dynamic Model

$T_{F}=k_{F}\left(\theta_{T R}-x_{V E H} / r_{F}\right)+b_{F}\left(\omega_{T R}-v_{V E H} / r_{F}\right)$

$T_{R}=k_{R}\left(\theta_{E M} \tau_{G B}-x_{V E H} / r_{R}\right)+b_{R}\left(\omega_{E M} \tau_{G B}-v_{V E H} / r_{R}\right)$

where $\dot{x}_{V E H}=v_{V E H}$ and $\dot{\theta}_{*}=\omega_{*}$.

\section{Decoupled Drivability Control}

The control strategy can be developed after expressing the dynamic equations using a proper state-space model. Define the following state vector:

$$
x=\left[\begin{array}{lllllll}
\omega_{I C E} & \omega_{T R} & \omega_{E M} & v_{V E H} & \theta_{T R} & \theta_{E M} & x_{V E H}
\end{array}\right]^{T}
$$

The input torques of a 3-DOF system form the control vector: 


$$
u=\left[\begin{array}{lll}
T_{I C E} & T_{I S A} & T_{E M}
\end{array}\right]^{T}
$$

The system given by (16a-d) can be re-written as

$$
M \dot{x}=f(x)+B u+\Gamma
$$

where $\Gamma$ is the vector of lumped uncertainties and disturbance torques, and

$$
B=\left[\begin{array}{ccc}
1 & 1 & 0 \\
0 & 0 & 0 \\
0 & 0 & 1 \\
\hline 0_{4 \times 3}
\end{array}\right] ; M=\operatorname{diag}\left\{\begin{array}{lllllll}
I C E & J_{T R} & J_{E M} & m_{V E H} & 1 & 1 & 1
\end{array}\right\}
$$

along with the following mechanical constraints imposed by the actuators and states:

$$
\begin{gathered}
0 \leq T_{I C E} \leq T_{I C E, M A X} \\
T_{E M, M I N} \leq T_{E M} \leq T_{E M, M A X} ; T_{I S A, M I N} \leq T_{I S A} \leq T_{I S A, M A X} \\
0 \leq \omega_{I C E} \leq \omega_{I C E, M A X} ; \omega_{E M, M I N} \leq \omega_{E M} \leq \omega_{E M, M A X}
\end{gathered}
$$

The instantaneous power demand, $P_{I N S T}$, is another hard constraint that should be respected by the controller. It can be written in the following compact form

$$
P_{I N S T}=x^{T} B u
$$

Analysis of (18) reveals that the drivability control can be treated separately from the equivalent fuel consumption control $\left(u_{E C M S}\right)$. Using the null control directions of the power constraint [9]

$$
\operatorname{Ker}\left(x^{T} B\right)=\operatorname{span}\left\{\left[\begin{array}{c}
1 \\
-1 \\
0
\end{array}\right],\left[\begin{array}{c}
0 \\
x_{3} \\
x_{1}
\end{array}\right]\right\}
$$

enables the choice of the following drivability control vector

$$
u_{v}(x)=\left[\begin{array}{lll}
0 & x_{3} & -x_{1}
\end{array}\right]^{T}
$$

Thus, if $u_{E C M S}$ is a solution of (18) then

$$
u=u_{E C M S}+v u_{v}
$$

also satisfies the power demand, where $v \in \mathbb{R}$. Barbarisi [9] also treated SOC control as an individual component, hence entirely decoupling SOC control from fuel management and drivability controls. Note that the additional drivability control input does not affect the instantaneous power of the vehicle which is determined by the equivalent fuel consumption control strategy. Following section describes an optimal control solution to obtain $v$.

\section{Drivability Metrics and Optimal Control}

The optimal control design is based on minimizing a single or a set of drivability metrics and the control effort. Various definitions of drivability have been proposed and a survey of these metrics can be found in [8].
Combining one or more drivability metrics a cost function can be defined as

$$
J=\int_{0}^{\infty}\left[(g(x, t))^{2}+\rho v^{2}\right] d t
$$

where $g(x, t)$ is the preferred drivability metric(s) and $\rho>0$ is a weighting factor. An infinite horizon, gain scheduled LQR design requires the dynamics (17) to be linearized at the operating points

$$
\begin{gathered}
\delta \dot{x}=A_{v} \delta x+B_{v} \delta v \\
A_{v}=M^{-1} \frac{\delta f(x)}{\delta x}+M^{-1} B \frac{\delta u_{v}}{\delta x} \\
B_{v}=M^{-1} B u_{v}
\end{gathered}
$$

Instead of having the controller run in real-time, the cost function is computed on a discrete grid of states, and for each grid point the controllability of the system is verified and the steady-state Riccati equation is solved for P:

$$
A_{v}^{T} P+P A_{v}-\frac{1}{\rho} P B_{v} B_{v}{ }^{T} P+Q=0
$$

The resulting controller is

$$
\delta v=-K(x) \delta x
$$

where $K(x)=(1 / \rho) B_{v}{ }^{T} P(x)$. The control gains, $K(x)$, are computed offline and a look-up table is generated using an array of potential values of the state variables.

\section{REFERENCES}

[1] C. C. Lin, J. M. Kang, J. W. Grizzle, and H. Peng, "Energy management strategy for parallel hybrid electric truck," in Proc. American Control Conference, 2001, pp. 2878-2883.

[2] M. Salman, N. J. Schouten, and N. A. Kheir, "Control strategies for parallel hybrid vehicles," in Proc. American Control Conference, Chicago, IL, 2000, pp. 524-528.

[3] B. M. Baumann, G. N. Washington, B. C. Glenn, and G. Rizzoni, "Mechatronic design and control of hybrid electric vehicles," IEEE/ASME Trans. on Mechatronics, vol. 5, no. 1, pp. 58-72, 2000.

[4] G. Paganelli, M. Tateno, A. Brahma, G. Rizzoni, and Y. Guezennec "Control development for a hybrid-electric sport-utility vehicle: Strategy implementation and field test results," in Proc. American Control Conference, Arlington, VA, 2001, pp. 5064-5069.

[5] G. Paganelli, G. Ercole, A. Brahma, Y. Guezennec, and G. Rizzoni, "General supervisory control policy for the energy optimization of chargesustaining hybrid electric vehicles," JSAE Review, vol. 22, pp. 511-518, 2001.

[6] P. Pisu, C. Musardo, B. Staccia, and G. Rizzoni. "A Comparative Study of Supervisory Control Strategies for Hybrid Electric Vehicles". IMECE 2004, 13-19 November 2004, Anaheim, CA.

[7] P. Pisu, G. Rizzoni, and E. Calo'. "Control Strategies for Parallel Hybrid Electric Vehicles”. IFAC'04, Salerno, Italy, 19-23 April 2004, pp.508-513.

[8] Xi Wei, P. Pisu, G. Rizzoni, and S. Yurkovich. "Dynamic Modeling of Hybrid Electric Drivetrain for Fuel Economy, Performance and Drivability Evaluations". 2003 ASME International Mechanical Engineering Congress \& Exposition, Washington, DC, 16-21 November 2003.

[9] O. Barbarisi, E. R. Westervelt, G. Rizzoni, and F. Vasca, "Power management decoupling control for a hybrid electric vehicle". Submitted to CDC'05. 